\title{
Insights of the dental calculi microbiome of pre-Columbian inhabitants from Puerto Rico
}

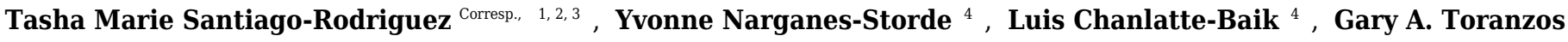 \\ 5 , Raul J. Cano ${ }^{1,3,6}$ \\ ${ }^{1}$ Center for Applications in Biotechnology, California Polytechnic State University - San Luis Obispo, San Luis Obispo, California, United States \\ 2 Biology Deparment, California Polytechnic State University - San Luis Obispo, San Luis Obispo, California, United States \\ 3 Institute for Life Science Entrepreneurship, ATCC-Center for Translational Microbiology, Union, NJ, United States \\ 4 Center for Archaeological Investigations, University of Puerto Rico, San Juan, PR \\ 5 Biology Department, University of Puerto Rico, San Juan, PR \\ 6 Biology, California Polytechnic State University - San Luis Obispo, San Luis Obispo, California, United States \\ Corresponding Author: Tasha Marie Santiago-Rodriguez \\ Email address: tsantiagoro@gmail.com
}

Background. The study of ancient microorganisms in mineralized dental plaque or calculi is providing insights of microbial evolution, as well as lifestyles and disease states of extinct cultures; yet, little is still known about the oral microbial community structure and function of pre-Columbian Caribbean cultures. In the present study, we investigated the dental calculi microbiome and predicted function of one of these cultures, known as the Saladoid. The Saladoids were horticulturalists that emphasized root-crop production. Fruits, as well as small marine and terrestrial animals were also part of the Saladoid diet.

Methods. Dental calculi samples were recovered from the archaeological site of Sorcé, in the municipal island of Vieques, Puerto Rico, characterized using $16 \mathrm{~S}$ rRNA gene high-throughput sequencing, and compared to the microbiome of previously characterized coprolites of the same culture, as well modern plaque, saliva and stool microbiomes available from the Human Microbiome Project.

Results. Actinobacteria, Proteobacteria and Firmicutes comprised the majority of the Saladoid dental calculi microbiome. The Saladoid dental calculi microbiome was distinct when compared to those of modern saliva and dental plaque, but showed the presence of common inhabitants of modern oral cavities including Streptococcus sp., Veillonella dispar and Rothia mucilaginosa. Cell motility, signal transduction and biosynthesis of other secondary metabolites may be unique features of the Saladoid microbiome.

Discussion. Results suggest that the Saladoid dental calculi microbiome structure and function may possibly reflect a horticulturalist lifestyle and distinct dietary habits. Results also open the opportunity to further elucidate oral disease states in extinct Caribbean cultures and extinct indigenous cultures with similar lifestyles. 


\section{Insights of the dental calculi microbiome of pre-Columbian inhabitants from}

2

3 Tasha M. Santiago-Rodriguez ${ }^{1,2,3^{*}}$, Yvonne M. Narganes-Storde ${ }^{4}$, Luis Chanlatte-Baik ${ }^{4 *}$, Gary

$5 \quad{ }^{1}$ Center for Applications in Biotechnology, California Polytechnic State University, San Luis

\section{Puerto Rico}

Obispo, California, United States; ${ }^{2}$ Biology Department, California Polytechnic State University,

San Luis Obispo, California, United States; ${ }^{3}$ Institute for Life Science Entrepreneurship, ATCCCenter for Translational Microbiology, Union, NJ, United States; ${ }^{4}$ Center for Archaeological Investigations, University of Puerto Rico, San Juan, PR; ${ }^{5}$ Biology Department, University of Puerto Rico, San Juan, PR

*Corresponding author: Tasha M. Santiago-Rodriguez; email: tsantiagoro@gmail.com; Phone: (787)-236-0059

**Deceased.

\section{Short title: Oral microbiome of Saladoids}

\section{ABSTRACT}

Background. The study of ancient microorganisms in mineralized dental plaque or calculi is providing insights of microbial evolution, as well as lifestyles and disease states of extinct cultures; yet, little is still known about the oral microbial community structure and function of pre-Columbian Caribbean cultures. In the present study, we investigated the dental calculi microbiome and predicted function of one of these cultures, known as the Saladoid. The 
24 Saladoids were horticulturalists that emphasized root-crop production. Fruits, as well as small

25 marine and terrestrial animals were also part of the Saladoid diet.

Methods. Dental calculi samples were recovered from the archaeological site of Sorcé, in the municipal island of Vieques, Puerto Rico, characterized using 16S rRNA gene high-throughput sequencing, and compared to the microbiome of previously characterized coprolites of the same culture, as well modern plaque, saliva and stool microbiomes available from the Human Microbiome Project.

Results. Actinobacteria, Proteobacteria and Firmicutes comprised the majority of the Saladoid dental calculi microbiome. The Saladoid dental calculi microbiome was distinct when compared to those of modern saliva and dental plaque, but showed the presence of common inhabitants of modern oral cavities including Streptococcus sp., Veillonella dispar and Rothia mucilaginosa. Cell motility, signal transduction and biosynthesis of other secondary metabolites may be unique features of the Saladoid microbiome.

Discussion. Results suggest that the Saladoid dental calculi microbiome structure and function may possibly reflect a horticulturalist lifestyle and distinct dietary habits. Results also open the opportunity to further elucidate oral disease states in extinct Caribbean cultures and extinct indigenous cultures with similar lifestyles. 


\section{INTRODUCTION}

48

49

64

Paleomicrobiology or the study of ancient microorganisms is providing insights into microbial evolution, the lifestyles of extinct cultures, as well as ancient diseases. Mummified gut remains (Ubaldi, Luciani et al. 1998, Cano, Tiefenbrunner et al. 2000, Santiago-Rodriguez, Fornaciari et al. 2015), coprolites (Tito, Knights et al. 2012, Santiago-Rodriguez, NarganesStorde et al. 2013, Cano, Rivera-Perez et al. 2014), bones (Yang, Eng et al. 1998) and dental calculi (Adler, Dobney et al. 2013, Warinner, Rodrigues et al. 2014, Warinner, Speller et al. 2015, Ziesemer, Mann et al. 2015) are known to harbor ancient microbial DNA. Dental calculi, particularly, is known to be commonly recovered from archaeological excavations across diverse geographical regions, and studies have shown that it is an important bacterial reservoir (Vandermeersch, Arensburg et al. 1994, Arensburg 1996, Preus, Marvik et al. 2011). Previous paleomicrobiology studies relied heavily on techniques such as microscopy and amplification of genes from particular bacterial species using the Polymerase Chain Reaction (PCR) (Pääbo, Higuchi et al. 1989, Cano and Borucki 1995, Pap, Tillier et al. 1995, Willerslev and Cooper 2005). Recent advances in high-throughput sequencing and bioinfomatic tools have enabled the study of bacterial communities by simultaneously amplifying and sequencing the $16 \mathrm{~S}$ ribosomal RNA (16S rRNA) gene (Caporaso, Kuczynski et al. 2010). bacterial species belonging to the Actinobacteria, Bacteroidetes, Firmicutes and Proteobacteria (Dewhirst, Chen et al. 2010). Unlike the human gut microbiome, little is still known about the potential effect that lifestyles, dietary habits and oral hygiene may exert on the oral microbiome (Adler, Dobney et al. 2013, Clemente, Pehrsson et al. 2015). A previous study characterizing the 
oral microbiome of individuals from three distinct geographical regions found profound bacterial taxonomic differences that may be attributed to culture (Li, Quinque et al. 2014). Another study found that the oral microbiome of uncontacted Amerindians is distinct and more diverse compared to subjects with westernized lifestyles (Clemente, Pehrsson et al. 2015). Similarly, a study characterizing the gut microbiome of hunter-gatherers from South America showed that it is different and more diverse compared to that of westernized cultures (Obregon-Tito, Tito et al. 2015). These studies indicate that modern, isolated societies have distinct microbiomes compared to modern westernized societies, and that these may better resemble the ancestral or original state of the human microbiome.

Microbial characterization of ancient human societies may potentially provide direct insights into the ancestral state of the oral microbiome (Warinner, Speller et al. 2015). Ancient human oral microbiomes have been studied through dental calculi, which have been found to be dominated by Actinobacteria, Firmicutes, Proteobacteria and Bacteroidetes, and also harbor oral pathogens (Warinner, Rodrigues et al. 2014). Previous studies have characterized the oral microbiome of ancient European cultures (Adler, Dobney et al. 2013, Warinner, Rodrigues et al. 2014). Results have shown that changes in dietary habits associated with specific time periods affect the structure and function of the human oral microbiome (Adler, Dobney et al. 2013). PreColumbian cultures also had a significant impact on modern western societies, and characterization of their oral microbiome may also provide insights of the ancestral state of the human oral microbiome throughout part of human history (Ziesemer, Mann et al. 2015). It is feasible to hypothesize that the recovered dental calculi may resemble taxonomic and functional profiles of modern dental plaque, and also possibly reflect pre-Columbian lifestyles. 
93

Archaeological evidence suggests that pre-Columbian cultures came in close contact with

95

96

97

98 each other, suggesting long-distance migrations possibly due to knowledge in seafaring (Curet 2005). The arrangement of the Caribbean archipelago may have facilitated the migration of preColumbian cultures from diverse regions across the Americas (Fig 1A). Migration throughout the Caribbean resulted in the settlement and development of a diverse mosaic of cultures. Puerto Rico, particularly, was an important site of ancient human migrations in the Caribbean, representing the longest continuous span of occupation in the Antilles (Curet 2005). Archaeological evidence in Puerto Rico suggest two major pre-Columbian migrations, that included the Archaic people and the Saladoids (Siegel 2005, Siegel 2010). The Saladoids migrated from South America (lower Orinoco River) and established at the site of Sorcé in the municipal island of Vieques, Puerto Rico around 180 B.C., and the main island of Puerto Rico by 430 B.C. While a major hypothesis suggests that the Saladoid culture was divided in two subseries, the Huecan and the Cedrosan Saladoid, an alternate hypothesis suggests that each of the subseries were unique and distinct cultures (Siegel 2005, Ramos 2010). The alternate hypothesis is mainly supported by differences in semi-precious stone ornaments, and ceramic paintings and decorations, the latter which still remain the primary analytical units of preColumbian Caribbean cultures. Based on the alternative hypothesis, the Saladoids co-inhabited with the Huecoids in Sorcé for over 1,000 years (5 A.D. to 1170 A.D.) (Siegel 2010). Archaeological and isotope evidence have suggested that the Saladoids relied on terrestrial resources and brought to Puerto Rico a developed horticultural economy, which may have been modified with interactions with Archaic groups (Siegel 2005). The Saladoid diet included manioc or yuca, sweet potatoes, yautia, squashes, maize, nuts, as well as fruits such as soursop, 
116 papaya, guava and passion fruit (Siegel 2005). Proteins were mainly acquired from the ingestion

117 of fish, crustaceans, bivalves, sea turtles, birds and small terrestrial mammals (Storde 1982, Baik

118 2013). While food preparation methods remain largely unknown (Siegel 2005), thoroughly

119 boiling of food items, such as tubers, prior to consumption would have been a feasible practice.

120 Food boiling may be consistent with the absence of nucleic acids from food items, such as

121 manioc, in ancient human samples.

122

Recently, microbial community analyses of coprolites found in Saladoid deposits from

124 Sorcé have shown marked differences when compared to coprolites found in Huecoid deposits

from the same site, as well as modern Amazonian cultures, suggesting that the gut microbiome may reflect culture-specific dietary habits (Santiago-Rodriguez, Narganes-Storde et al. 2013,

127 Cano, Rivera-Perez et al. 2014). Teeth with dental calculi from the Saladoid culture at Sorcé have also been recovered, representing an opportunity to provide insights into their oral microbiome. Unfortunately, Huecoid human remains, including teeth, have not been recovered from the archaeological site of Sorcé and were not included in the present study; yet, the previously characterized coprolite microbiomes of the Saladoid culture, as well as the microbiomes of supragingival and subginvival plaque, saliva, stool and soil from the archaeological site of Sorcé have been included in the present study. It is hypothesized that the oral microbiome of the Saladoids may be distinct from modern individuals, possibly due to differences in culture, lifestyles, dietary habits, and lack of oral hygiene; therefore, the aims of the present study were to characterize the taxonomic and predicted functional categories of

137 Saladoid dental calculi using 16S rRNA gene high-throughput sequencing, and compare them to 138 the microbiomes of modern oral, gut and soil from the archaeological site of Sorcé. 
MATERIALS AND METHODS

141

142

143

144

145

146

147

148

149

150

151

152

153

154

155

156

157

158

159

160

\section{Archaeological site and sample description}

The archaeological excavation site was located at the Sorcé Estate in La Hueca, Vieques,

Puerto Rico (Fig 1B). The excavation was directed by archeologists Luis Chanlatte-Baik and

Yvonne Narganes-Storde from the Center for Archaeological Investigations at the University of Puerto Rico. A total of 12 loose dental samples were included in the present study and were excavated from the Saladoid deposit YTA-2 at different depths. Three dental calculi samples were recovered from teeth attached to bone fragments that enabled gender or age determination by forensic archeologist Dr. Edwin Crespo from the University of Puerto Rico (Table 1). Age-at death and sex were determined by the application of morphological and quantitative methods as described in (Bass 1971, Krogman and Işcan 1986, Scheuer and Black 2000). Teeth for this study were acquired with all necessary field permits complying with all relevant regulations for the collection of skeletal remains from archeological sites. Repository information, including the nomenclature for precise identification containing geographical location, excavation site and archaeological depth are described in Table $1 .{ }^{14} \mathrm{C}$ dating was conducted by Teledyne Isotopes (Westwood, NJ) and BETA Analytic, Inc. (Miami, FL, USA) using standard methods. Most recovered teeth samples correspond to adult subjects, with the exception of sample H6, which was identified as an infant, showing moderate to severe wear and carious lesions. Fig $\mathbf{1 C}$ shows examples of the teeth included in the present study.

\section{Dental calculi recovery and DNA extraction}



Biotechnology at the California Polytechnic State University, San Luis Obispo, dedicated for ancient DNA analyses. Hoods were sterilized with UV-radiation at least $15 \mathrm{~min}$ before and after every use. Dental calculi were separated from teeth using a dental scalar kindly provided by Dr. bleach between samples. Protective clothing and gloves were worn at all times, and were also 167 changed between samples. The recovered dental calculi were then placed in sterilized tubes and pulverized using custom-made micropestles. Micropestles were sterilized using UV-radiation, and bleach between samples. A mixture of supragingival and subgingival calculi was obtained from each tooth as it was not possible to make a clear distinction. In order to reduce environmental contamination, DNA extraction was performed in dedicated class II hood, where the calculi was also recovered, using the PowerSoil DNA Isolation Kit following manufacturer's instructions with the following modifications: (i) the PowerBead tube was placed in a Qbiogene Fast Prep Instrument (Carlsbad, CA) at $4.5 \mathrm{~m} / \mathrm{s}$ for $30 \mathrm{~s}$ and then centrifuged at $10,000 \times \mathrm{g}$ for 30 $\mathrm{s}$; and (ii) incubation periods at $-20^{\circ} \mathrm{C}$ for $15 \min$ (Mo Bio Laboratories, Carlsbad, CA, USA). The PowerSoil DNA Isolation Kit can recover DNA fragments as short as 60 bp (Qiagen technical support personal communication). All necessary equipment for DNA extraction,

178 including micropipets, was sterilized before and after every use with a $10 \%$ bleach solution and $17970 \%$ ethanol. Soil from the archaeological site of Sorcé surrounding the maxillary fragment of 180 sample M8 (Table 1) was recovered as a control for further analyses. A blank control was also included throughout the DNA extraction, PCR amplification and sequencing as described below. 

using DNAaway and UV-radiation to eliminate cross-contamination with modern samples.

Template manipulations are handled in separate hoods that are sterilized before and after every manipulation using DNAaway and UV-radiation. Negative PCR controls were included in all amplification reactions, showing no amplification. The 16S rRNA gene V4 variable region was amplified using the universal PCR primers 515f(GTGCCAGCMGCCGCGGTAA)/806r

192

(GGACTACHVGGGTWTCTAAT), as described previously (Caporaso, Lauber et al. 2012).

PCR amplifications were conducted using a single step 30 cycle PCR using the HotStarTaq Plus Master Mix Kit (Qiagen, USA) under the following conditions: $94{ }^{\circ} \mathrm{C}$ for $3 \mathrm{~min}$, followed by 28 cycles of $94{ }^{\circ} \mathrm{C}$ for 30 seconds, $53{ }^{\circ} \mathrm{C}$ for 40 seconds and $72{ }^{\circ} \mathrm{C}$ for $1 \mathrm{~min}$, after which a final elongation step at $72{ }^{\circ} \mathrm{C}$ for 5 min was performed. Two PCR amplifications following the conditions described above were performed due to the limiting starting material and to obtain higher yields to continue with library preparation. After amplification, PCR products were checked in $2 \%$ agarose gel to determine the success of amplification and the relative intensity of the bands. All amplicon products from each sample were mixed or pooled after tagmentation in equal concentrations and purified using Agencourt AMPure beads (Agencourt Bioscience Corporation, MA, USA). The pooled and purified PCR products were used to prepare the DNA library following Illumina MiSeq DNA library preparation protocol using the MiSeq reagent kit v2 (2X150 bp) on a MiSeq following the manufacturer's guidelines. Preprocessing of reads including removal of chimeras and singletons was performed using proprietary tools from 
206 MRDNA. Phred scores were checked using the FastQC tool

207 http://www.bioinformatics.babraham.ac.uk/projects/fastqc/

208

209

210

16S rRNA gene analyses

211

Due to data availability, modern oral and gut samples used for comparison were obtained

212 from the Human Microbiome Project (HMP) (http:/hmpdacc.org/). Data correspond to overall

213 healthy individuals, age 18-40, from the USA. Further information about these subjects is

214 available at http://www.ncbi.nlm.nih.gov/projects/gap/cgi-

215 bin/study.cgi?study_id=phs000228.v3.p1. Stool samples corresponded to an Amazonian culture.

216 These samples were chosen over USA individuals because that may better resemble that of the

217 Saladoid culture due to similarities in lifestyles. All 16S rRNA gene sequence analyses were

218 performed using the Quantitative Insights Into Microbial Ecology (Qiime) (Caporaso, Kuczynski

219 et al. 2010). Reads were assigned to samples based on their corresponding barcode using

220 split_libraries.py with default filtering parameters. Operational taxonomic units (OTUs) were

221 selected using the pick_closed_reference_otus.py workflow in Qiime because different regions

222 of the 16S rRNA gene were being characterized. 16S rRNA taxonomy was defined by $97 \%$

223 similarity to reference sequences. OTU biom files were created for the modern and ancient oral

224 and gut microbiome sequences, and checked using biom summarize-table. OTU biom files were

225 also created separately for the blank control and soil from the archeological site of Sorcé, and

226 checked using biom summarize-table. OTUs present in the blank control (Data Set S1) and soil

227 from the archaeological site of Sorcé (Data Set S2) were removed from all the samples using the

228 workflow described in http://qiime.org/tutorials/filtering_contamination_otus.html to eliminate 
229 the potential contamination effect of laboratory reagents and post-mortem environment to the

230 results. All subsequent analyses were also performed with unfiltered soil and blank control

231 OTUs.

232

233

Bayesian Source Tracker or SourceTracker analyses were then performed to identify

234

235

236

237

238

239

240

241

242

243

244

245

246

247

248

249

250

251

The lowest sequence count was 245 after removing from the dental calculi samples OTUs corresponding to the blank control and soil from the archaeological site of Sorcé; thus, data were rarefied to 245 sequences to minimize the effect of disparate sequence number to the results.

Rarefaction allows the calculation of species richness through the construction of rarefaction curves. Taxonomy, as well as alpha- and beta-diversity plots were determined using the core_diversity_analyses.py workflow. Alpha diversities, including chao1 and observed OTUs were computed from the average of ten iterations using the collate_alpha.py workflow (Chao 1984, Gotelli and Colwell 2001). Statistical analyses were performed using the alpha_compare.py workflow with default parameters. Since subsampled reads for each comparison were used, a Bonferroni correction was applied. 2D plots were constructed as 
252 described http://qiime.org/scripts/make_2d_plots.html. Group significance analyses were

253 performed using the group_signficance.py script in Qiime with default parameters, including

254 Kruskal-Wallis as the statistical test and 1,000 permutations. The test compares OTU frequencies

255 between sample groups to see if these are differentially represented. The category considered in

256 this analysis was sample type including dental calculi, dental calculi from teeth attached to bone,

257 supragingival plaque, subgingival plaque, saliva, stool, coprolite and soil from the archaeological

258 site of Sorcé. The core microbiome between the dental calculi and modern oral microbiomes (i.e.

259 supragingival and subgingival plaque, as well as saliva) was computed using the

260 compute_core_microbiome.py after filtering soil and blank OTUs in order to identify shared

261 OTUs between ancient and modern oral samples. The script identifies the core OTUs in a biom

262 table.Minimum fraction for core was set to 0.25 or $25 \%$. Analyses stopped at 0.40 or $40 \%$,

263 indicating that no OTUs were shared in over $40 \%$ of the samples.

264

265

Prediction of functional categories based on 16S rRNA gene data was performed using

the package Phylogenetic Investigation of Communities by Reconstruction of Unobserved States

267 (PICRUSt) available online at https://huttenhower.sph.harvard.edu/galaxy/ (Langille, Zaneveld

et al. 2013). Functional profiles predicted at level 2 were visualized using a heatmap that was constructed using the function heatmap. 2 available in the $\mathrm{R}$ package gplots. The relative abundances of the functional profiles were also visualized using Linear Discriminatory Analysis 
275

276

277

278

279

280

281

282

283

284

285

286

287

288

289

290

291

292

293

294

295

296

297

\section{RESULTS}

\section{S rRNA gene diversity of dental calculi}

Fifteen dental calculi samples from the Saladoid culture were recovered from the archaeological site of Sorcé, Puerto Rico, and the DNA characterized using 16S rRNA gene high-throughput sequencing (Table 1). An average of $692 \pm 429$ dental calculi sequences with an average size of 272 bp (after quality filtering including chimeras and singletons removal), were analyzed. Average Phred score was 40. An average of 5,097 $\pm 1,007$ (supragingival plaque); 4,094 \pm 372 (subgingival plaque); 5,686 \pm 1369 (saliva); 2,640,130 $\pm 548,433$ (stool); $48,100 \pm 32,461$ (coprolites); and 22,945 (soil from the archaeological site of Sorcé) sequences were also analyzed. OTUs corresponding to a blank control and soil from the archaeological site of Sorcé are included in Data Sets S1 and S2, respectively, and were filtered from the dental calculi OTUs, as described above. Prior any further analyses, we performed SourceTracker analyses to identify possible sources of contamination, and modern microbiomes that could potentially share resemblance with the dental calculi microbiomes. Results showed that $>99.0 \%$ of the dental calculi sequences did not resemble any of the modern or ancient human microbiomes (shown as unknown in grey) (Fig S1 Panels A-O). SourceTracker analyses were also performed prior filtering soil and blank control OTUs. Results showed a percentage of OTUs in the dental calculi samples that resemble soil and blank controls prior filtering (Fig S1 Panels A-O). The dental calculi microbiomes were then compared to those of coprolites from the same culture, and modern dental plaque, saliva, stool and soil from the archaeological site of Sorcé. Data were rarefied to 245 sequences (given that this was the lowest sequence count after filtering OTUs from soil and blank control) in order to minimize the effect of disparate sequence number on the results (Table 1 and Table S1). Samples reached or were close to reach a plateau 
298 when data were rarefied, as demonstrated with the chaol (Fig S2A) and observed OTUs (Fig

299 S2B) rarefaction plots. Rarefaction curves of chaol (Fig S2C) and observed OTUs (Fig S2D)

300 indices prior filtering OTUs corresponding to soil and blank control from the dental calculi

301 samples are also shown. Two different alpha diversity indices including chao1 (Fig 2A) and

302 observed OTUs (Fig 2B) were plotted after filtering OTUs corresponding to soil and blank

303 control. Chao1 (Fig S3A) and observed OTUs (Fig S3B) indices prior filtering soil and blank

304 control OTUs are also shown. chao1 (Table S2) and observed OTUs (Table S3) values

305 exhibiting statistically significant differences are highlighted in bold. Values shown in Tables S2

306 and S3 correspond to samples after filtering soil and blank control OTUs. Analyses were also

307 performed with unfiltered soil and blank control OTUs, showing differing p-values in both chao1

308 (Table S4) and observed OTUs (Table S5) when compared to filtered soil and blank control 309 OTUs.

310

311

Principal Coordinate Analyses (PCoA) plots were constructed to visualize beta-

312

313

314

315

316

317

318

319

320

diversity indices of the modern and ancient oral and gut microbiomes, as well as that of soil from the archaeological site of Sorcé (Fig 3). Results show clustering based on sample type. Analyses were also performed with unfiltered soil and blank control OTUs, showing a separation based on sample type. Interestingly, the soil sample clustered closely to stool samples from Amazonians

(Fig S4). On average, Actinobacteria (14.7\%), Proteobacteria (28.4 \%) and Firmicutes (26.5 \%) comprised the majority of the Saladoid dental calculi microbiome at the phylum level. Notably, the taxonomic composition of the dental calculi recovered from bone that enabled gender or age determination exhibited variations in these relative abundances. For instance, sample H6, identified as an infant, had $55.7 \%$ of the OTUs classified as Bacteroidetes, from which $43.2 \%$ 
321 where Bacteroides sp. Actinobacteria comprised an average of $29.4 \%, 12.7 \%, 2.6 \%, 2.3 \%$ and

$32263.0 \%$ of the bacterial communities in modern supragingival and subgingival plaque, saliva and

323 stool, and coprolites, respectively (Fig 4). The majority of the modern supragingival plaque

324 microbiome was comprised by Firmicutes (27.3\%), Bacteroidetes (17.6 \%), Fusobacteria (13.5

$325 \%)$ and Proteobacteria (10.3\%). The majority of the modern subgingival plaque microbiome was

326 also composed of the same phyla as supragingival plaque, but in differing proportions,

327 depending on the subject (Fig 4). Notably, the microbiome of soil from the archaeological site of

328 Sorcé was mostly comprised by Firmicutes (57.3\%) and Actinobacteria (34.8\%) (Fig 4). Taxa

329 identified in the blank control are shown in Data Set S3. Taxonomy analyses at the phylum level

330 with unfiltered soil and blank control OTUs showed differing proportions of Proteobacteria and

331 Firmicutes (Fig S5). Taxonomic analyses at the genus level after filtering soil and blank control

332 OTUs enabled the identification of 869 taxonomic groups, where 345 could not be classified at

333 the genus level (Data Set S4). Genera including Corynebacterium sp., Lactobacillus sp., Rothia

334 sp., Staphylococcus sp., Streptococcus sp., and Treponema sp. were identified in both ancient

335 and modern oral microbiomes. Taxonomic classification at the genus level prior filtering soil and

336 blank OTUs showed 840 taxonomic groups, where 325 could not be classified at the genus level

337 (Data Set S5). A number of unclassified taxonomic groups that were present in soil were also

338 identified in both ancient and modern microbiomes.

339

Group significance analyses after filtering soil and blank control OTUs showed a number

341 of unique OTUs in the dental calculi microbiomes that were not identified in the modern and

342 ancient gut or oral microbiomes (Data Set S6). There were also a number of shared OTUs

343 between modern oral and dental calculi microbiomes including, but not limited to Streptococcus 
344 (OTU 1082294), Veillonella dispar (OTU 962249), Rothia mucilaginosa (OTU 1017181) and

345 Porphyromonas sp. (OTU 4301737) (Table 2 and Data Set S6). Group significance analyses

346 prior filtering soil and blank OTUs showed OTUs shared between soil and the dental calculi

347 samples (Data Set S7). Interestingly, OTUs 172063 (Bacillus sp.), 961009 (Acinetobacter

348 johnsonii), 1088265 (Propionibacterium acnes) and 3453734 (Acinetobacter sp.) were identified

349 in the soil, dental calculi and Amazonian stool samples. Core microbiome analyses of the dental

350 calculi samples after filtering soil and blank OTUs, and supragingival and subgingival plaque, as

351 well as saliva showed Rothia mucilaginosa in both ancient (Data Set S8) and modern oral (Data

352 Set S9) microbiomes.

353

354 Predicted functional categories

$355 \quad$ Functional categories in ancient and modern oral and gut microbiomes were predicted

356 using PICRUSt. In principle, PICRUSt does not predict functional categories from 16S rRNA

357 gene data, since the originating organisms are not being detected, but rather the "bleed over" of

358 putative pathways from the underlying catalog due to cross-annotation of gene families,

359 computationally detected sequence homology, or very rarely from sequence misclassification.

360 Most often this is due to conserved genes being annotated to those pathways in KEGG and other

361 databases, although the actual underlying gene family is something more basic (e.g. cell cycle or

362 structural) (Curtis Huttenhower personal communication). The relative abundances of the

363 functional categories at level 2 were visualized using heatmap plots (Fig 5). Overall, the modern

364 gut and oral microbiomes clustered separately from the coprolites, dental calculi and soil from

365 the archaeological site of Sorcé (Fig 5). In the cluster including the modern microbiomes, there

366 was a separation based on biogeographical site. In the cluster including the ancient microbiomes, 
367 there was, in turn, a separation between the coprolites and dental calculi microbiomes (Fig 5).

368 Functional categories were also predicted prior filtering soil and blank OTUs. Results also

369 showed a separation of the dental calculi samples from the modern microbiomes, but expectedly,

370 functional profiles exhibited differing proportions (Fig S6).

371

372

Differences in the functional categories predicted using PICRUSt were visualized using

373 LEfSe plots. Dental calculi microbiomes were characterized by functional categories associated

374 with cell motility, signal transduction and biosynthesis of other secondary metabolites. Modern

375 oral microbiomes were characterized by functional categories associated with replication and

376 repair; translation; nucleotide metabolism; metabolism of cofactors and vitamins; glycan

377 biosynthesis and metabolism; folding, sorting and degradation; cell growth and death; as well as

378 signaling molecules and interaction. The microbiome of soil from the archaeological site of

379 Sorcé was characterized by metabolism of other amino acids (Fig 6). Unique functional

380 categories of the coprolites and modern gut microbiomes are also shown in Fig 6. LEfSe

381

382

383 analyses of unfiltered soil and blank control OTUs showed that cell motility and signal transduction were still unique features of the dental calculi samples. Categories unique to each sample type were similar prior and after filtering soil and blank control OTUs (Fig S7).

\section{DISCUSSION}

Contamination of human samples by the post-mortem environment is an issue when working with ancient oral samples that have been in direct contact with soil as microbes may penetrate the dentition. This issue has previously been raised for dental calculi found in archaeological sites in the Virgin Islands (Ziesemer, Mann et al. 2015). One possible approach to 
390

391

392

393

394

395

396

397

398

400

401

402

403

404

405

406

407

408

409

410

411

412

authenticate ancient microbiomes is utilizing Bayesian Source Tracker analyses (Tito, Knights et al. 2012, Warinner, Rodrigues et al. 2014, Santiago-Rodriguez, Fornaciari et al. 2015). In the present study, Saladoid's dental calculi microbiomes did not resemble the modern or ancient microbiomes included in the analyses. SourceTracker analyses showed a number of OTUs in the dental calculi samples resembling soil from the archaeological site of Sorcé and a blank control; thus, these OTUs were filtered from the dental calculi sequences prior main analyses. Filtering OTUs corresponding to soil from the archaeological site of Sorcé and a blank control possibly increased the potential of analyzing sequences associated exclusively with dental calculi. A previous study showed that even after decontamination of the exterior of dental calculi, a number of soil sequences may still be detected; therefore, filtering soil OTUs may give a better resolution of the authentic dental calculi microbiome (Ziesemer, Mann et al. 2015). The present, as well as previous studies have found bacterial DNA in commercially available extraction kits (Glassing, Dowd et al. 2016); therefore, it is of importance to include a blank control in sequencing reactions that could be used to filter potential contaminating OTUs prior further analyses.

While a previous study characterizing the dental calculi microbiomes of ancient European cultures showed that these resembled dental plaque microbiomes of people of European ancestry (Warinner, Rodrigues et al. 2014), our data showed that the dental calculi microbiome of the Saladoid culture is taxonomically different at the phylum level and generally more diverse compared to modern dental plaque. It has been argued that this is due to contamination with soil microbes (Warinner, Rodrigues et al. 2014). While this is a possibility that is often visited by other researchers in the field of ancient DNA, it should also be considered that differences in dietary habits and hygiene practices may have an impact on the taxonomic 
413 structure of the human oral microbiome. Studies characterizing the dental plaque microbiome of

414 modern isolated and industrialized cultures with differences in ancestry, dietary habits and oral

415 hygiene are needed to provide better insights of the evolution of the human oral microbiome

416 throughout part of human history. For instance, a recent study characterizing the saliva

417 microbiome of the Tsimane, indigenous people from the Amazon, showed that the Firmicutes,

418 Bacteroidetes, Actinobacteria and Proteobacteria comprised the majority of the bacterial taxa in

419 adults. The salivary microbiome of Tsimane children was characterized by not resembling that of

420 their mothers and having a higher relative proportion of Firmicutes (Han, Martin et al. 2016).

421 Although researchers sampled saliva, results resemble some our findings. This resemblance may

422 be due to the Tsimane diets consisting primarily of cultivated carbohydrates and fish, similar to

423 the diet of the Saladoid culture from Puerto Rico. We also found evidence of the presence of

424 bacteria known to inhabit the oral cavity of modern subjects in the Saladoid's dental calculi,

425 including Streptococcus sp., Porphyromonas sp., and Gemellaceae sp., supporting previous

426 findings that bacterial members of the human oral microbiome are ubiquitous across diverse

427 geographical regions and time periods (Adler, Dobney et al. 2013, Warinner, Rodrigues et al.

428 2014, Clemente, Pehrsson et al. 2015).

429

Even though the DNA extraction and sequencing methods differed, our data are

consistent with another study characterizing the dental calculi microbiome of a pre-Columbian

432 Caribbean culture inhabiting the Virgin Islands, where Actinobacteria comprised a large

433 proportion of the bacterial taxa (Ziesemer, Mann et al. 2015). This same study showed that

434 tropical climates may not have the same degree of ancient bacterial DNA preservation as colder

435 climates. Given that these samples have been in contact with soil for extended periods of time, it 
436 is expected to find 16S rRNA gene sequences matching soil microbiomes, even after external

437 decontamination of the dental calculi; yet, one advantage of the bioinformatic tools developed

438 for $16 \mathrm{~S}$ rRNA gene analyses is that reads corresponding to soil contaminants can be filtered.

439 Bioinformatic tools available to discriminate potential sources of contamination, such as

440 SourceTracker, may not conclusively indicate that results in the present study were influenced by

441 soil contamination. While bioinformatic analyses do not truly support that the presence of

442 Actinobacteria is due to contamination with the post-mortem environment, it is feasible to

443 hypothesize that the OTUs present in the dental calculi samples reflect, to some extent, dietary

444 habits of the Saladoid culture. It is hypothesized that the Saladoid diet was mainly composed of

445 root crops and fruits, whose residues are known to get entrapped in dental calculi (Mickleburgh

446 and Pagán-Jiménez 2012). It is also possible that intake of root crops and fruits contaminated

447 with soil may have had some impact in the bacterial taxa being detected in the dental calculi

448 samples. Differences in lifestyles and dietary habits may explain some of the lack of similarity

449 between the Saladoid and ancient European oral microbiomes characterized previously, even

450 when both of these ancient cultures lacked oral hygienic conditions. In addition, some members

451 of the Actinobacteria, including Actinomyces have been associated with dental caries in modern

452 individuals. Detection of Actinomyces DNA in the Saladoid is intriguing as it supports

453 observations that pre-Columbian cultures from the Caribbean also suffered from oral diseases

454 (Chalmers, Oh et al. 2015, Ziesemer, Mann et al. 2015). Our data may also be consistent with a

455 previous study suggesting that dental caries in ancient human populations may have resulted

456 from the intake of fermentable carbohydrates (Adler, Dobney et al. 2013), as there is

457 archaeological evidence suggesting that the Saladoid ingested fermentable carbohydrates

458 (Widstrom, Carr et al. 1987, Humphrey, De Groote et al. 2014) that are part of the root crops. 
Results may have also been influenced by the amplified 16S rRNA gene variable region.

461

462

463

464

465

466

467

468

469

470

471

472

473

474

475

476

477

478

479

480

481

Recent evidence suggests that amplification of different 16S rRNA gene variable regions may also have profound effects on ancient microbiome studies due to PCR amplification biases (Ziesemer, Mann et al. 2015). This study also suggested that amplification of the V4 region may be impractical for ancient DNA studies; however, $<300$ bp is still within what would be considered the norm in the field of ancient DNA (Drancourt, Aboudharam et al. 1998, von Wurmb-Schwark, Schwark et al. 2004, Roberts and Ingham 2008, Paéibo 2012). In addition, the V4 region has been better characterized and has shown to have a better phylogenetic resolution (Yang, Wang et al. 2016) than other 16S rRNA gene variable regions.

Due to the small number of recovered sequences after filtering soil from the archeological site of Sorcé and blank control OTUs, special consideration should be taken when interpreting results from the prediction of functional categories in the dental calculi samples. In addition, while PICRUSt was utilized to predict functional profiles based on 16S rRNA gene data, which has shown to work more efficiently with stool microbiomes, results may still add valuable information regarding the potential effect of culture, lifestyle, dietary habits, oral hygiene and disease states to the ancestral state of human oral microbiomes. Ancient and modern oral microbiomes showed shared functional profiles, suggesting that certain predicted functional pathways may be ubiquitous to the human oral microbiome. While taphonomic conditions may have influenced the predicted functional profiles of the dental calculi included in the present study, it is also feasible to hypothesize that some of the predicted functional profiles were altered due to differences in culture, lifestyle, dietary habits, oral hygiene and disease 
482 states, which are known to affect the community structure and function of modern human oral

483 microbiomes (Zarco, Vess et al. 2012).

484

485 CONCLUSIONS

486

Skepticism is met when studying the oral microbiomes of ancient Caribbean cultures as

487

there is always the risk that soil bacteria may have penetrated the dentine; thus, the reliability of 488 the microbiome structure and function of dental calculi excavated from tropical regions may be challenged. Tools such as Bayesian SourceTracker analyses may be used to authenticate ancient microbiomes by providing insights into the contribution of possible contaminating sources, including soil from the archaeological site, as well as reagents. Our data may provide insights of the oral microbiome of the Saladoid culture from Sorcé, Puerto Rico, although a deeper sequencing depth is needed. Comparative studies would ideally include plaque and saliva samples from modern indigenous individuals, but access to this type of samples is challenging. Given that most human microbiomes studies have been performed with subjects from the US limits our ability to interpret some of the results. Bacteria present in the oral cavities of modern subjects were also present in the dental calculi microbiome of the Saladoid culture. Advantages and disadvantages exist when using $16 \mathrm{~S}$ rRNA gene high-throughput sequencing to characterize ancient human oral microbiomes.

\section{ACKNOWLEDGEMENTS}

502

We would like to acknowledge Mark Brolaski for providing reagents for the DNA extraction, and Christina Warinner for providing valuable insights in microbiome methods to study ancient

504 dental calculi. 
505

506

507

508

509

510

511

512

513

514

515

516

517

518

519

520

521

522

523

524

525

526

527

\section{REFERENCES}

Adler, C. J., K. Dobney, L. S. Weyrich, J. Kaidonis, A. W. Walker, W. Haak, C. J. Bradshaw, G. Townsend, A. Soltysiak, K. W. Alt, J. Parkhill and A. Cooper (2013). "Sequencing ancient calcified dental plaque shows changes in oral microbiota with dietary shifts of the Neolithic and Industrial revolutions." Nat Genet 45(4): 450-455, 455e451.

Arensburg, B. (1996). "Ancient dental calculus and diet." Human Evolution 11(2): 139-145.

Baik, L. A. C. (2013). "Huecoid Culture and the Antillean Agroalfarero (Farmer-Potter) Period." The Oxford Handbook of Caribbean Archaeology: 171.

Bass, W. M. (1971). "Human osteology: a laboratory and field manual of the human skeleton."

Cano, R. J. and M. K. Borucki (1995). "Revival and identification of bacterial spores in 25-to 40million-year-old Dominican amber." Science 268(5213): 1060.

Cano, R. J., J. Rivera-Perez, G. A. Toranzos, T. M. Santiago-Rodriguez, Y. M. Narganes-Storde, L. Chanlatte-Baik, E. Garcia-Roldan, L. Bunkley-Williams and S. E. Massey (2014).

"Paleomicrobiology: revealing fecal microbiomes of ancient indigenous cultures." PLoS One 9(9): e106833.

Cano, R. J., F. Tiefenbrunner, M. Ubaldi, C. Del Cueto, S. Luciani, T. Cox, P. Orkand, K. H. Kunzel and F. Rollo (2000). "Sequence analysis of bacterial DNA in the colon and stomach of the Tyrolean Iceman." Am J Phys Anthropol 112(3): 297-309.

Caporaso, J. G., J. Kuczynski, J. Stombaugh, K. Bittinger, F. D. Bushman, E. K. Costello, N. Fierer, A. G. Peña, J. K. Goodrich and J. I. Gordon (2010). "QIIME allows analysis of highthroughput community sequencing data." Nature methods 7(5): 335-336.

Caporaso, J. G., J. Kuczynski, J. Stombaugh, K. Bittinger, F. D. Bushman, E. K. Costello, N. Fierer, A. G. Pena, J. K. Goodrich, J. I. Gordon, G. A. Huttley, S. T. Kelley, D. Knights, J. E. 
528 Koenig, R. E. Ley, C. A. Lozupone, D. McDonald, B. D. Muegge, M. Pirrung, J. Reeder, J. R.

529 Sevinsky, P. J. Turnbaugh, W. A. Walters, J. Widmann, T. Yatsunenko, J. Zaneveld and R.

530 Knight (2010). "QIIME allows analysis of high-throughput community sequencing data." Nat

531 Methods 7(5): 335-336.

532 Caporaso, J. G., C. L. Lauber, W. A. Walters, D. Berg-Lyons, J. Huntley, N. Fierer, S. M.

533 Owens, J. Betley, L. Fraser, M. Bauer, N. Gormley, J. A. Gilbert, G. Smith and R. Knight

534 (2012). "Ultra-high-throughput microbial community analysis on the Illumina HiSeq and MiSeq 535 platforms." ISME J 6(8): 1621-1624.

536 Chalmers, N. I., K. Oh, C. V. Hughes, N. Pradhan, E. Kanasi, Y. Ehrlich, F. E. Dewhirst and A.

537 C. Tanner (2015). "Pulp and plaque microbiotas of children with severe early childhood caries."

538 J Oral Microbiol 7: 25951.

539 Chao, A. (1984). "Nonparametric estimation of the number of classes in a population."

540 Scandinavian Journal of statistics: 265-270.

541 Clemente, J. C., E. C. Pehrsson, M. J. Blaser, K. Sandhu, Z. Gao, B. Wang, M. Magris, G.

542 Hidalgo, M. Contreras, O. Noya-Alarcon, O. Lander, J. McDonald, M. Cox, J. Walter, P. L. Oh,

543 J. F. Ruiz, S. Rodriguez, N. Shen, S. J. Song, J. Metcalf, R. Knight, G. Dantas and M. G.

544 Dominguez-Bello (2015). "The microbiome of uncontacted Amerindians." Sci Adv 1(3).

545 Curet, L. A. (2005). Caribbean paleodemography: population, culture history, and sociopolitical 546 processes in ancient Puerto Rico, University of Alabama Press.

547 Dewhirst, F. E., T. Chen, J. Izard, B. J. Paster, A. C. Tanner, W. H. Yu, A. Lakshmanan and W. 548 G. Wade (2010). "The human oral microbiome." J Bacteriol 192(19): 5002-5017. 
549 Drancourt, M., G. Aboudharam, M. Signoli, O. Dutour and D. Raoult (1998). "Detection of 400-

550 year-old Yersinia pestis DNA in human dental pulp: an approach to the diagnosis of ancient

551 septicemia." Proceedings of the National Academy of Sciences 95(21): 12637-12640.

552 Glassing, A., S. E. Dowd, S. Galandiuk, B. Davis and R. J. Chiodini (2016). "Inherent bacterial

553 DNA contamination of extraction and sequencing reagents may affect interpretation of

554 microbiota in low bacterial biomass samples." Gut pathogens 8(1): 1.

555 Gotelli, N. J. and R. K. Colwell (2001). "Quantifying biodiversity: procedures and pitfalls in the

556 measurement and comparison of species richness." Ecology letters 4(4): 379-391.

557 Han, C. S., M. A. Martin, A. E. Dichosa, A. R. Daughton, S. Frietze, H. Kaplan, M. D. Gurven

558 and J. Alcock (2016). "Salivary microbiomes of indigenous Tsimane mothers and infants are

559 distinct despite frequent premastication." PeerJ 4: e2660.

560 Humphrey, L. T., I. De Groote, J. Morales, N. Barton, S. Collcutt, C. B. Ramsey and A.

561 Bouzouggar (2014). "Earliest evidence for caries and exploitation of starchy plant foods in

562 Pleistocene hunter-gatherers from Morocco." Proceedings of the National Academy of Sciences

563 111(3): 954-959.

564 Knights, D., J. Kuczynski, E. S. Charlson, J. Zaneveld, M. C. Mozer, R. G. Collman, F. D.

565 Bushman, R. Knight and S. T. Kelley (2011). "Bayesian community-wide culture-independent

566 microbial source tracking." Nat Methods 8(9): 761-763.

567 Krogman, W. M. and M. Işcan (1986). "The human skeleton in forensic science." CC Thomas:

568 Springfield.

569 Langille, M. G., J. Zaneveld, J. G. Caporaso, D. McDonald, D. Knights, J. A. Reyes, J. C.

570 Clemente, D. E. Burkepile, R. L. Vega Thurber, R. Knight, R. G. Beiko and C. Huttenhower 
571 (2013). "Predictive functional profiling of microbial communities using 16S rRNA marker gene

572 sequences." Nat Biotechnol 31(9): 814-821.

573 Li, J., D. Quinque, H. P. Horz, M. Li, M. Rzhetskaya, J. A. Raff, M. G. Hayes and M. Stoneking

574 (2014). "Comparative analysis of the human saliva microbiome from different climate zones:

575 Alaska, Germany, and Africa." BMC Microbiol 14: 316.

576 Mickleburgh, H. L. and J. R. Pagán-Jiménez (2012). "New insights into the consumption of

577 maize and other food plants in the pre-Columbian Caribbean from starch grains trapped in

578 human dental calculus." Journal of Archaeological Science 39(7): 2468-2478.

579 Obregon-Tito, A. J., R. Y. Tito, J. Metcalf, K. Sankaranarayanan, J. C. Clemente, L. K. Ursell, Z.

580 Zech Xu, W. Van Treuren, R. Knight, P. M. Gaffney, P. Spicer, P. Lawson, L. Marin-Reyes, O.

581 Trujillo-Villarroel, M. Foster, E. Guija-Poma, L. Troncoso-Corzo, C. Warinner, A. T. Ozga and

582 C. M. Lewis (2015). "Subsistence strategies in traditional societies distinguish gut microbiomes."

583 Nat Commun 6: 6505.

584 Pääbo, S., R. G. Higuchi and A. C. Wilson (1989). "Ancient DNA and the polymerase chain

585 reaction: The emerging field of molecular archaeology (Minireview)." The Journal of biological

586 chemistry 264(17): 9709-9712.

587 Paéibo, S. (2012). "Amplifying ancient DNA." PCR protocols: a guide to methods and 588 applications: 159.

589 Pap, I., A.-M. Tillier, B. Arensburg, S. Weiner and M. Chech (1995). "First scanning electron

590 microscope analysis of dental calculus from European Neanderthals: Subalyuk,(Middle

591 Paleolithic, Hungary). Preliminary report." Bulletins et Mémoires de la Société d'anthropologie

592 de Paris 7(1): 69-72. 
593 Preus, H. R., O. J. Marvik, K. A. Selvig and P. Bennike (2011). "Ancient bacterial DNA (aDNA)

594 in dental calculus from archaeological human remains." Journal of Archaeological Science 595 38(8): 1827-1831.

596 Ramos, R. R. (2010). Rethinking Puerto Rican Precolonial History, University of Alabama Press. 597 Roberts, C. and S. Ingham (2008). "Using ancient DNA analysis in palaeopathology: a critical 598 analysis of published papers, with recommendations for future work." International Journal of 599 Osteoarchaeology 18(6): 600-613.

600 Santiago-Rodriguez, T. M., G. Fornaciari, S. Luciani, S. E. Dowd, G. A. Toranzos, I. Marota and 601 R. J. Cano (2015). "Gut Microbiome of an 11th Century A.D. Pre-Columbian Andean Mummy." 602 PLoS One 10(9): e0138135.

603 Santiago-Rodriguez, T. M., Y. M. Narganes-Storde, L. Chanlatte, E. Crespo-Torres, G. A. 604 Toranzos, R. Jimenez-Flores, A. Hamrick and R. J. Cano (2013). "Microbial communities in pre605 columbian coprolites." PLoS One 8(6): e65191.

606 Scheuer, L. and S. Black (2000). "Development and ageing of the juvenile skeleton." Human 607 osteology in archaeology and forensic science: 9-22.

608 Siegel, P. E. (2005). Ancient Borinquen: archaeology and ethnohistory of native Puerto Rico, 609 University of Alabama Press.

610 Siegel, P. E. (2010). "Continuity and change in the evolution of religion and political 611 organization on pre-Columbian Puerto Rico." Journal of Anthropological Archaeology 29(3): $612 \quad 302-326$.

613 Storde, Y. M. N. (1982). Vertebrate faunal remains from Sorce, Vieques, Puerto Rico. 
614 Tito, R. Y., D. Knights, J. Metcalf, A. J. Obregon-Tito, L. Cleeland, F. Najar, B. Roe, K.

615 Reinhard, K. Sobolik, S. Belknap, M. Foster, P. Spicer, R. Knight and C. M. Lewis, Jr. (2012).

616 "Insights from characterizing extinct human gut microbiomes." PLoS One 7(12): e51146.

617 Ubaldi, M., S. Luciani, I. Marota, G. Fornaciari, R. J. Cano and F. Rollo (1998). "Sequence

618 analysis of bacterial DNA in the colon of an Andean mummy." Am J Phys Anthropol 107(3):

$619 \quad 285-295$.

620 Vandermeersch, B., B. Arensburg, A.-M. Tillier, Y. Rak, S. Weiner, M. Spiers and E. Aspillaga

621 (1994). "Middle Palaeolithic dental bacteria from Kebara, Israel." Comptes rendus de l'Académie

622 des sciences. Série 2. Sciences de la terre et des planètes 319(6): 727-731.

623 von Wurmb-Schwark, N., T. Schwark, M. Harbeck and M. Oehmichen (2004). "A simple

624 duplex-PCR to evaluate the DNA quality of anthropological and forensic samples prior short 625 tandem repeat typing." Legal Medicine 6(2): 80-88.

626 Warinner, C., J. F. Rodrigues, R. Vyas, C. Trachsel, N. Shved, J. Grossmann, A. Radini, Y.

627 Hancock, R. Y. Tito, S. Fiddyment, C. Speller, J. Hendy, S. Charlton, H. U. Luder, D. C.

628 Salazar-Garcia, E. Eppler, R. Seiler, L. H. Hansen, J. A. Castruita, S. Barkow-Oesterreicher, K.

629 Y. Teoh, C. D. Kelstrup, J. V. Olsen, P. Nanni, T. Kawai, E. Willerslev, C. von Mering, C. M.

630 Lewis, Jr., M. J. Collins, M. T. Gilbert, F. Ruhli and E. Cappellini (2014). "Pathogens and host

631 immunity in the ancient human oral cavity." Nat Genet 46(4): 336-344.

632 Warinner, C., C. Speller and M. J. Collins (2015). "A new era in palaeomicrobiology: prospects

633 for ancient dental calculus as a long-term record of the human oral microbiome." Philos Trans R

634 Soc Lond B Biol Sci 370(1660): 20130376. 
635 Widstrom, N., M. Carr, M. Bagby and L. Black (1987). "Harvest methods for estimated ethanol 636 yields from relative fermentable carbohydrate accumulation in maize hybrids." Agronomy 637 Journal 79(4): 758-760.

638 Willerslev, E. and A. Cooper (2005). "Review paper. ancient dna." Proceedings of the Royal 639 Society of London B: Biological Sciences 272(1558): 3-16.

640 Yang, B., Y. Wang and P.-Y. Qian (2016). "Sensitivity and correlation of hypervariable regions 641 in 16S rRNA genes in phylogenetic analysis." BMC bioinformatics 17(1): 135.

642 Yang, D. Y., B. Eng, J. S. Waye, J. C. Dudar and S. R. Saunders (1998). "Technical note:

643 improved DNA extraction from ancient bones using silica-based spin columns." American 644 journal of physical anthropology 105(4): 539-543.

645 Zarco, M. F., T. J. Vess and G. S. Ginsburg (2012). "The oral microbiome in health and disease 646 and the potential impact on personalized dental medicine." Oral Dis 18(2): 109-120.

647 Ziesemer, K. A., A. E. Mann, K. Sankaranarayanan, H. Schroeder, A. T. Ozga, B. W. Brandt, E. 648 Zaura, A. Waters-Rist, M. Hoogland, D. C. Salazar-Garcia, M. Aldenderfer, C. Speller, J.

649 Hendy, D. A. Weston, S. J. MacDonald, G. H. Thomas, M. J. Collins, C. M. Lewis, C. Hofman 650 and C. Warinner (2015). "Intrinsic challenges in ancient microbiome reconstruction using 16S 651 rRNA gene amplification." Sci Rep 5: 16498.

652

653 Figure legends

654 Fig 1. Location and example of the samples obtained. Panel A shows the organization of the 655 Caribbean as a site for ancient human migrations. Vieques Island, which is part of the main 656 island of Puerto Rico, is highlighted by the red circle. Panel B shows the location of the 
657 archaeological site of Sorcé on Vieques. Panel C shows examples of the teeth recovered from the 658 archeological site of Sorcé.

659 Fig 2. Bar plots of alpha diversity indices. Bar plots representing the chao 1 (Panel A) and 660 observed OTUs (Panel B) indices for the bacterial taxonomy based on 16S rRNA gene of the 661 dental calculi, modern supragingival and subgingival plaque, saliva, coprolites, stool and soil 662 from the archaeological site of Sorcé microbiomes. Alpha diversity indices were computed from 663 the average of ten iterations using the collate_alpha.py workflow. Soil from the archeological 664 site of Sorcé and blank control OTUs were filtered from the dental calculi prior analyses.

665 Fig 3. Principal Coordinates Analysis (PCoA) 2D plots of ancient and modern oral and gut 666 microbiomes, as well as that of soil from the archaeological site of Sorcé. Dental calculi, dental 667 calculi of teeth attached to bones that enabled the identification of gender or age (Dental calculi 668 (Bone)), coprolites, stool, soil from the archaeological site of Sorcé, supragingival (grey) and 669 subgingival plaque (black) and saliva (white). Soil from the archeological site of Sorcé and blank 670 control OTUs were filtered from the dental calculi prior analyses.

671 Fig 4. Barplots representing the bacterial taxonomy based on 16S rRNA gene. Data are shown at 672 the phylum level for dental calculi, supragingival plaque, subgingival plaque, saliva, coprolites, 673 stool and soil from the archeological site of Sorcé. Soil from the archeological site of Sorcé and 674 blank control OTUs were filtered from the dental calculi prior analyses.

675 Fig 5. Heatmap of the normalized relative abundances of the predicted functional categories

676 (level 2) of the microbiomes of dental calculi, coprolites, supragingival and subgingival plaque, 677 saliva, stool and soil from the archeological site of Sorcé. Functional categories were predicted 678 using PICRUSt. Soil from the archeological site of Sorcé and blank control OTUs were filtered 679 prior analyses. 
680 Fig 6. Linear discriminatory analyses Effect size (LEfSe) plots of predicted functional categories

681 (level 2). Functional categories of dental calculi (ancient oral microbiomes), supragingival

682 plaque, subgingival plaque and saliva (modern oral microbiomes), coprolites (ancient gut

683 microbiomes), stool (modern gut microbiomes) and soil from the archeological site of Sorcé

684 (tropical soil) were predicted using PICRUSt. Soil from the archeological site of Sorcé and blank

685 control OTUs were filtered prior analyses.

\section{Supplementary Figure legends}

687 Fig S1. SourceTracker analyses of the dental calculi samples including D21 (Panel A), E19

688 (Panel B), E26 (Panel C), F20 (Panel D), G18 (Panel E), G21 (Panel F), G22 (Panel G), I19

689 (Panel H), I23A (Panel I), I23B (Panel J), I24A (Panel K), I24B (Panel L), F24 (Panel M), H6

690 (Panel N) and M8 (Panel O). Dental calculi microbiomes were compared to stool, coprolite,

691 saliva, soil from the archaeological site of Sorcé, subgingival plaque, and supragingival plaque

692 microbiomes. Dental calculi sequences not matching any of the microbiomes included were

693 classified as unknown. Figure shows results prior and after filtering soil and blank control OTUs

694 from the dental calculi.

695 Fig S2. Alphararefaction curves of chao1 (Panel A), and observed OTUs values (Panel B) after

696 filtering soil and blank control OTUs from the dental calculi, and chao1 (Panel C), and observed

697 OTUs (Panel D) values prior filtering soil and blank control OTUs. Samples included dental

698 calculi from loose teeth samples (yellow), dental calculi from teeth attached to bone (red),

699 supragingival plaque (pink), subgingival plaque (green), saliva (orange), coprolites (light blue),

700 stool (blue), soil from the archaeological site of Sorcé (brown), and blank control (purple).

701 Fig S3. Bar plots of alpha diversity indices. Bar plots representing the chao 1 (Panel A) and

702 observed OTUs (Panel B) indices for the bacterial taxonomy based on 16S rRNA gene of the 
703 dental calculi, modern supragingival and subgingival plaque, saliva, coprolites, stool and soil

704 from the archaeological site of Sorcé microbiomes. Alpha diversity indices were computed from

705 the average of ten iterations using the collate_alpha.py workflow. Soil and blank control OTUs

706 were not filtered from the dental calculi prior analyses.

707 Fig S4. Principal Coordinates Analysis (PCoA) 2D plots of ancient and modern oral and gut

708 microbiomes, as well as that of soil from the archaeological site of Sorcé. Dental calculi, dental

709 calculi of teeth attached to bones that enabled the identification of gender or age (Dental calculi

710 (Bone)), coprolites, stool, soil from the archaeological site of Sorcé, supragingival (grey) and

711 subgingival plaque (black), and saliva (white). Soil and blank control OTUs were not filtered

712 from the dental calculi prior analyses.

713 Fig S5. Barplots representing the bacterial taxonomy based on 16S rRNA gene. Data are shown

714 at the phylum level for dental calculi, supragingival plaque, subgingival plaque, saliva,

715 coprolites, stool, soil from the archeological site of Sorcé, and a blank control. Soil and blank

716 control OTUs were not filtered from the dental calculi prior analyses.

717 Fig S6. Heatmap of the relative abundances of the predicted functional categories (level 2) of

718 microbiomes of dental calculi, coprolites, supragingival and subgingival plaque, saliva, stool and

719 soil from the archeological site of Sorcé. Functional categories were predicted using PICRUSt.

720 Soil and blank control OTUs were not filtered from the dental calculi prior analyses.

721 Fig S7. Linear discriminatory analyses Effect size (LEfSe) plots of predicted functional

722 categories (level 2). Functional categories of dental calculi (ancient oral microbiomes),

723 supragingival plaque, subgingival plaque and saliva (modern oral microbiomes), coprolites

724 (ancient gut microbiomes), stool (modern gut microbiomes) and soil from the archeological site 
725 of Sorcé (tropical soil) were predicted using PICRUSt. Soil and blank control OTUs were not

726 filtered from the dental calculi prior analyses.

727 Supplementary Table legends

728 Table S1. Modern oral or stool microbiomes subjects'information. Sequences were downloaded 729 from the Human Microbiome Project (HMP).

730 Table S2. Chao1 statistical analyses. Statistically significant results are shown in bold. Soil and 731 blank control OTUs were filtered from the dental calculi prior analyses.

732 Table S3. Observed OTUs statistical analyses. Statistically significant results are shown in bold.

733 Soil and blank control OTUs were filtered from the dental calculi prior analyses.

734 Table S4. Chao1 statistical analyses. Statistically significant results are shown in bold. Soil and

735 blank control OTUs were not filtered from the dental calculi prior analyses.

736 Table S5. Observed OTUs statistical analyses. Statistically significant results are shown in bold.

737 Soil and blank control OTUs were not filtered from the dental calculi prior analyses.

738 Supplementary Data Sets legends

739 Data Set S1. OTU_table_Blank_Control.txt

740 Data Set S2. OTU_table_Soil.txt

741 Data Set S3. Taxa_found_in_Blank_Control.xls

742 Data Set S4. Dental_Calculi_Genus_Level_Filtered.xls

743 Data Set S5. Dental_Calculi_Genus_Level_Unfiltered.xls

744 Data Set S6. Group_Significance_Dental_Calculi_Filtered.xls

745 Data Set S7. Group_Significance_Dental_Calculi_Unfiltered.xls

746 Data Set S8. Core_OTUs_25_Dental_Calculi.txt

747 Data Set S9. Core_OTUs_25_Modern_Oral_Microbiome.txt 
Figure 1

Map Samples

Location and example of the samples obtained. Panel A shows the organization of the Caribbean as a site for ancient human migrations. Vieques Island, which is part of the main island of Puerto Rico, is highlighted by the red circle. Panel B shows the location of the archaeological site of Sorcé on Vieques. Panel C shows examples of the teeth recovered from the archeological site of Sorcé.

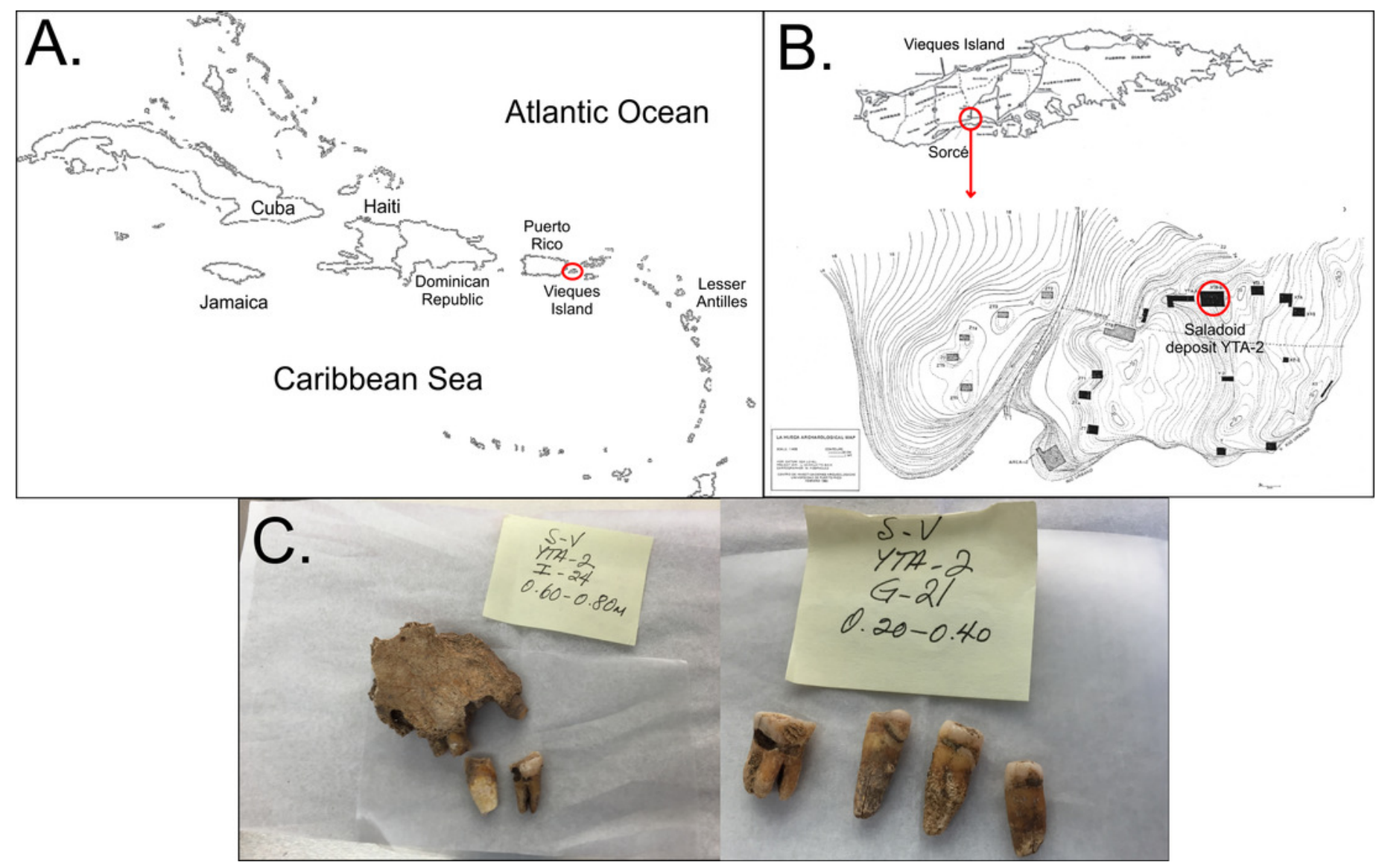




\section{Figure 2}

\section{Alpha Diversity Filtered}

Bar plots of alpha diversity indices. Bar plots representing the chao 1 (Panel A) and observed OTUs (Panel B) indices for the bacterial taxonomy based on 16S rRNA gene of the dental calculi, modern supragingival and subgingival plaque, saliva, coprolites, stool and soil from the archaeological site of Sorcé microbiomes. Alpha diversity indices were computed from the average of ten iterations using the collate_alpha.py workflow. Soil from the archeological site of Sorcé and blank control OTUs were filtered from the dental calculi prior analyses. 
A.

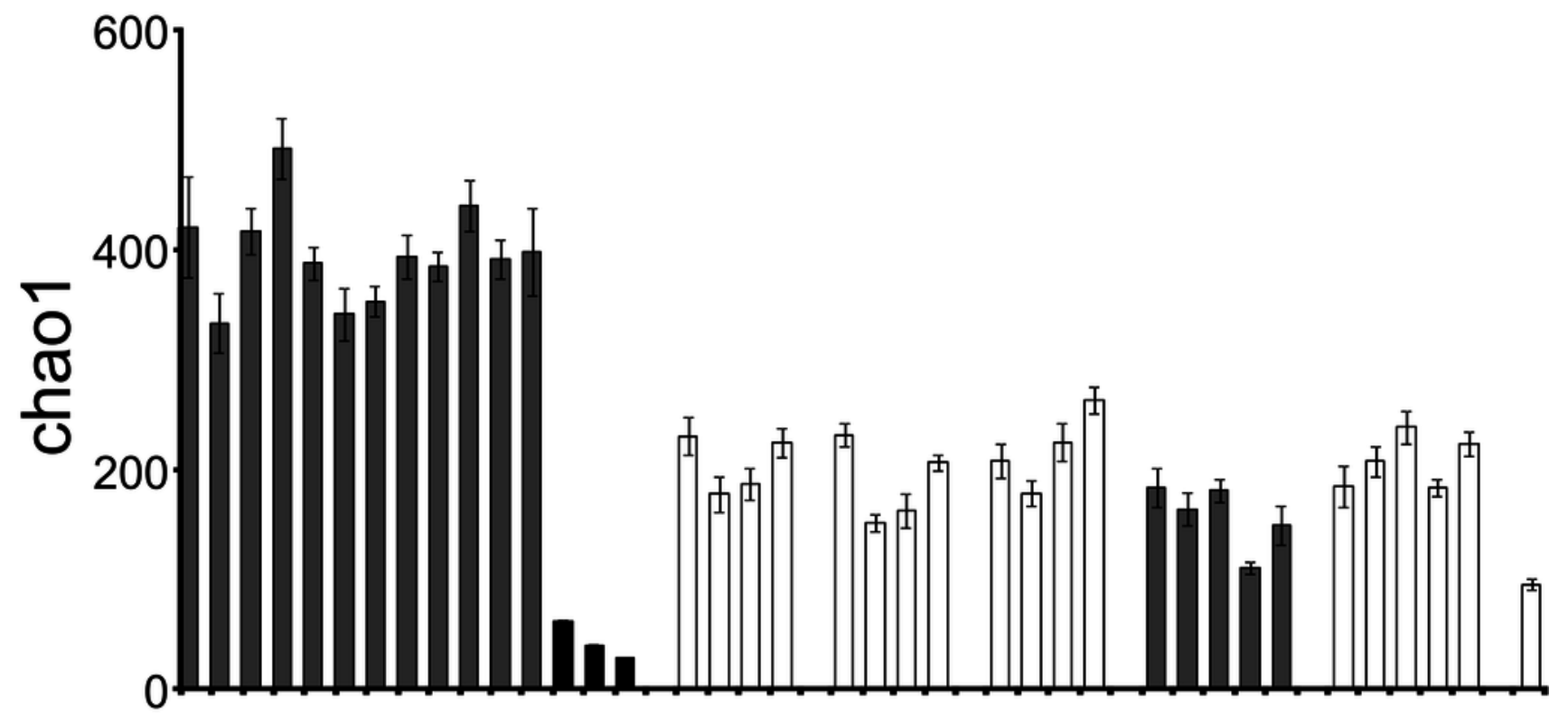

B.

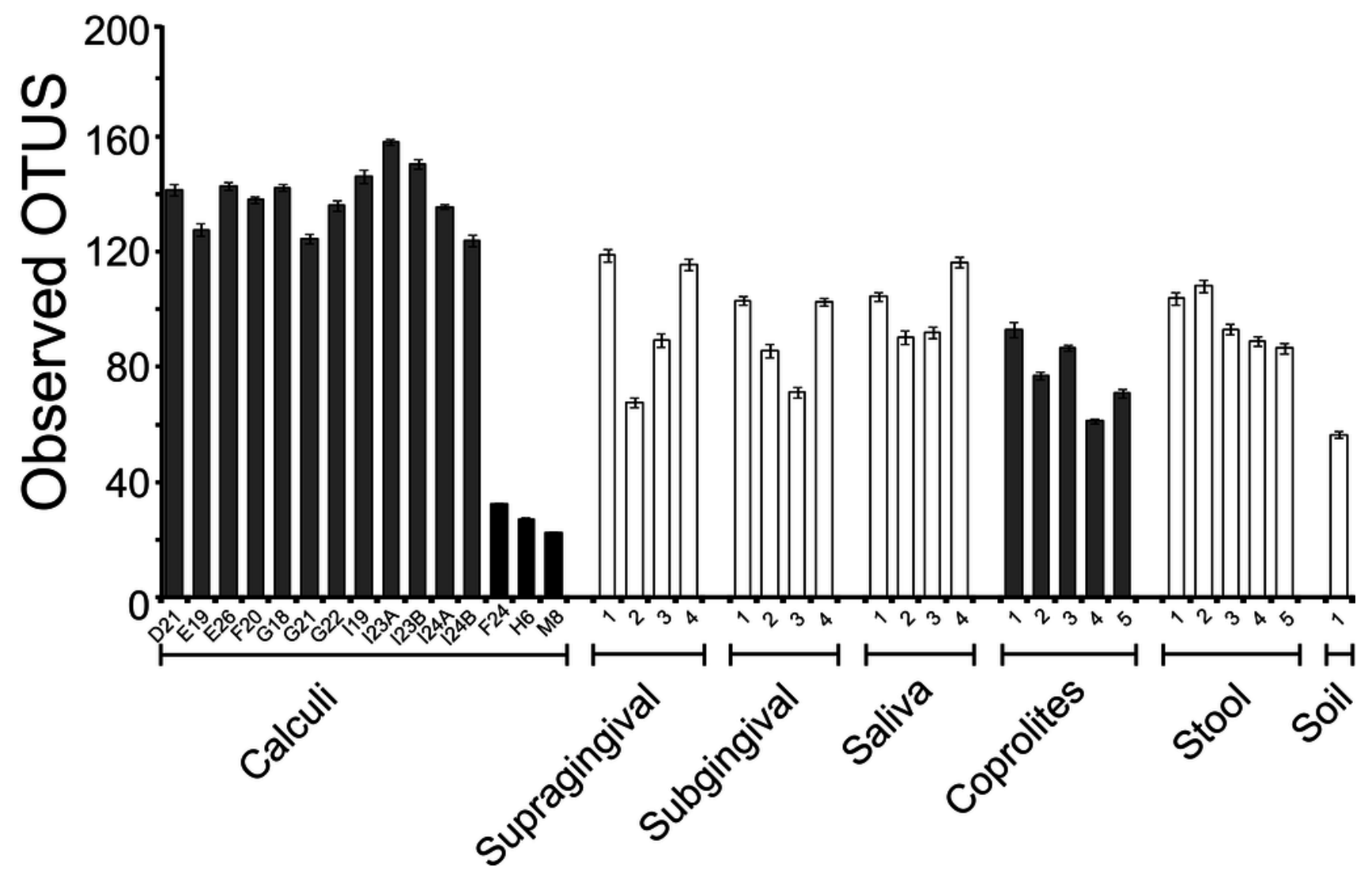




\section{Figure 3}

\section{PCoA Filtered}

Principal Coordinates Analysis (PCOA) 2D plots of ancient and modern oral and gut microbiomes, as well as that of soil from the archaeological site of Sorcé. Dental calculi, dental calculi of teeth attached to bones that enabled the identification of gender or age (Dental calculi (Bone)), coprolites, stool, soil from the archaeological site of Sorcé, supragingival (grey) and subgingival plaque (black) and saliva (white). Soil from the archeological site of Sorcé and blank control OTUs were filtered from the dental calculi prior analyses. 


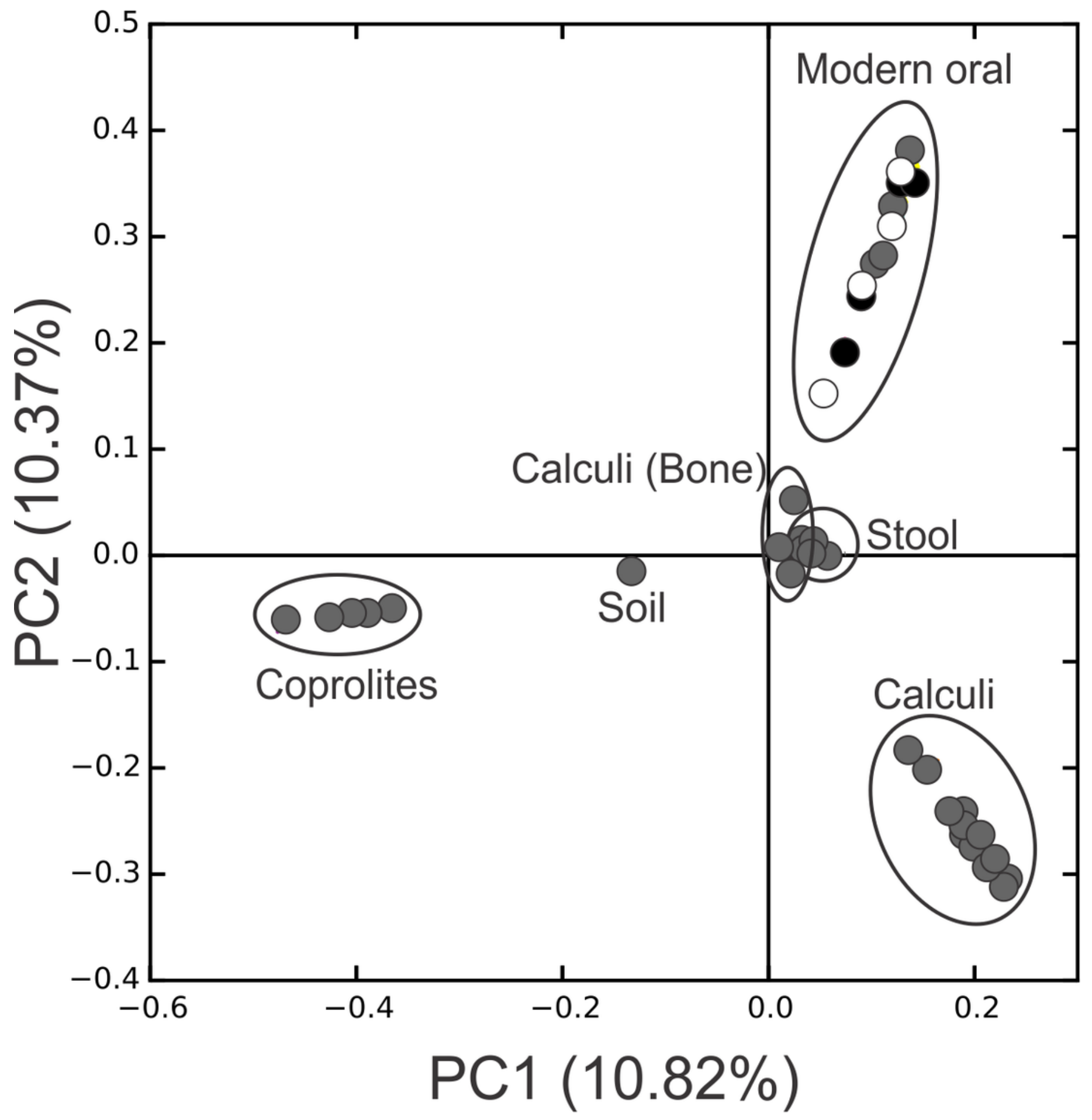


Figure 4

\section{Taxa Plots Filtered}

Barplots representing the bacterial taxonomy based on 16S rRNA gene. Data are shown at the phylum level for dental calculi, supragingival plaque, subgingival plaque, saliva, coprolites, stool and soil from the archeological site of Sorcé. Soil from the archeological site of Sorcé and blank control OTUs were filtered from the dental calculi prior analyses.

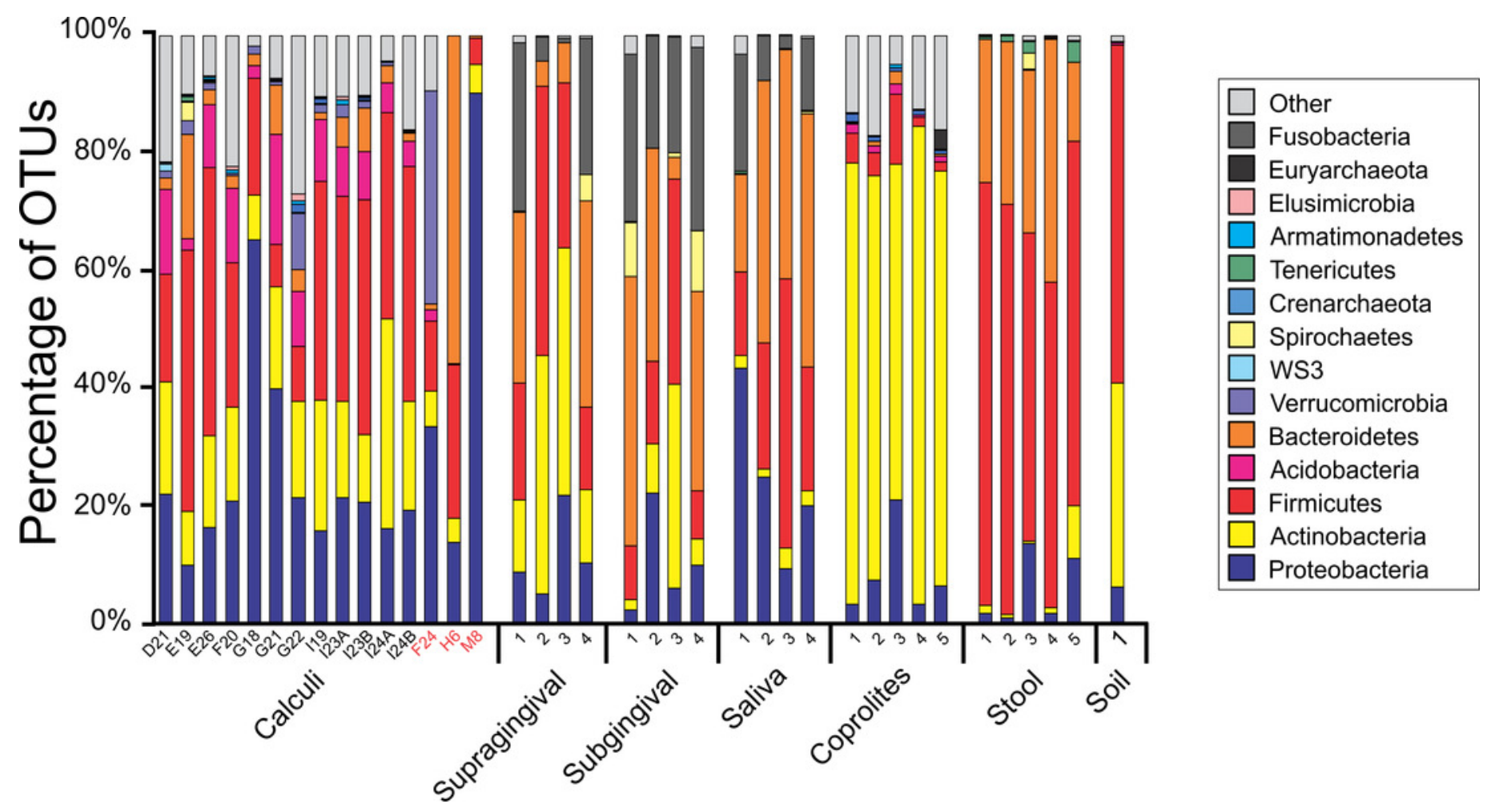




\section{Figure 5}

\section{PICRUSt}

Heatmap of the normalized relative abundances of the predicted functional categories (level

2) of the microbiomes of dental calculi, coprolites, supragingival and subgingival plaque,

saliva, stool and soil from the archeological site of Sorcé. Functional categories were predicted using PICRUSt. Soil from the archeological site of Sorcé and blank control OTUs were filtered prior analyses.

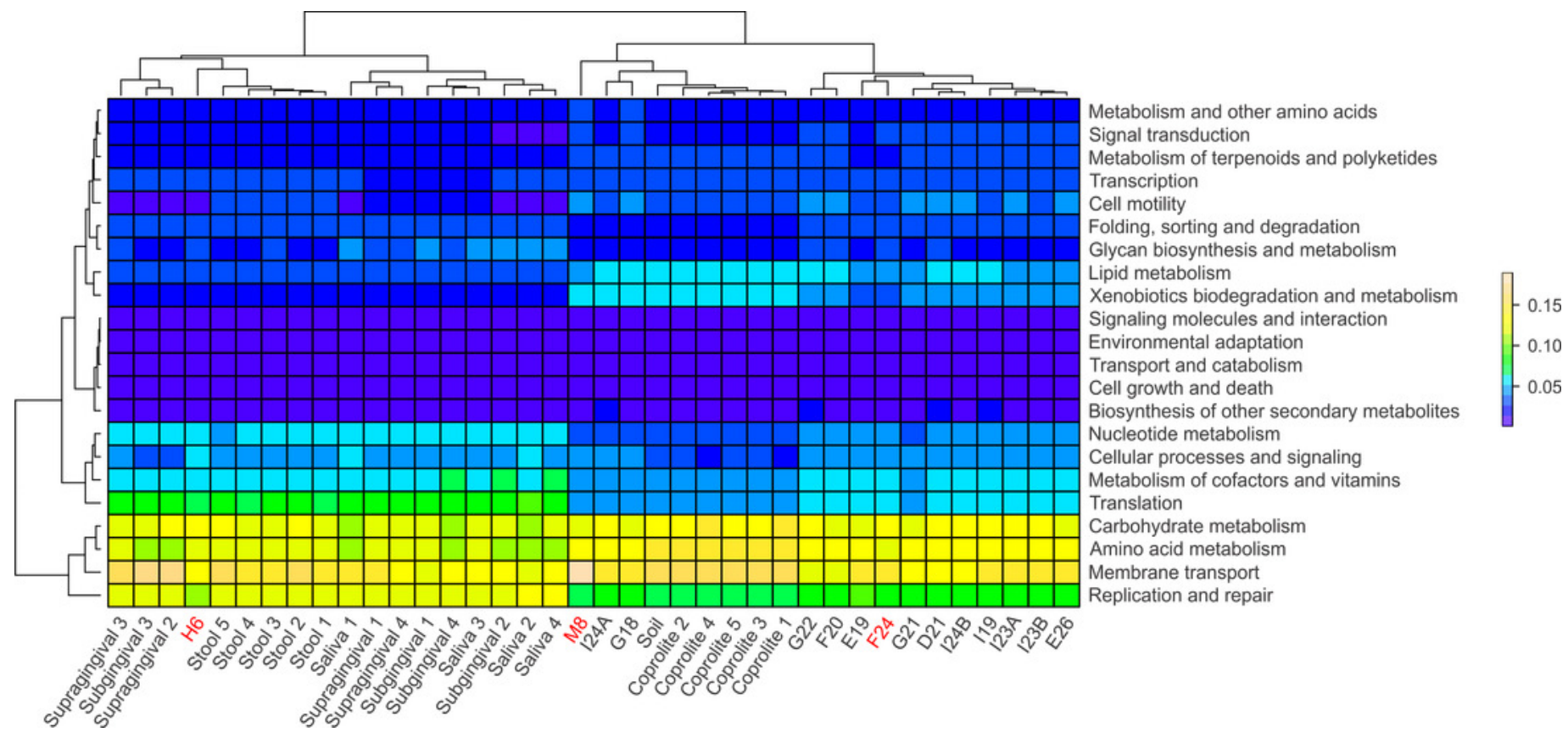




\section{Figure 6}

\section{LEfSe Filtered}

Linear discriminatory analyses Effect size (LEfSe) plots of predicted functional categories (level 2). Functional categories of dental calculi (ancient oral microbiomes), supragingival plaque, subgingival plaque and saliva (modern oral microbiomes), coprolites (ancient gut microbiomes), stool (modern gut microbiomes) and soil from the archeological site of Sorcé (tropical soil) were predicted using PICRUSt. Soil from the archeological site of Sorcé and blank control OTUs were filtered prior analyses.

\section{Ancient gut $\square$ Ancient oral $\square$ Modern gut $\square$ Modern oral $\square$ Tropical soil}

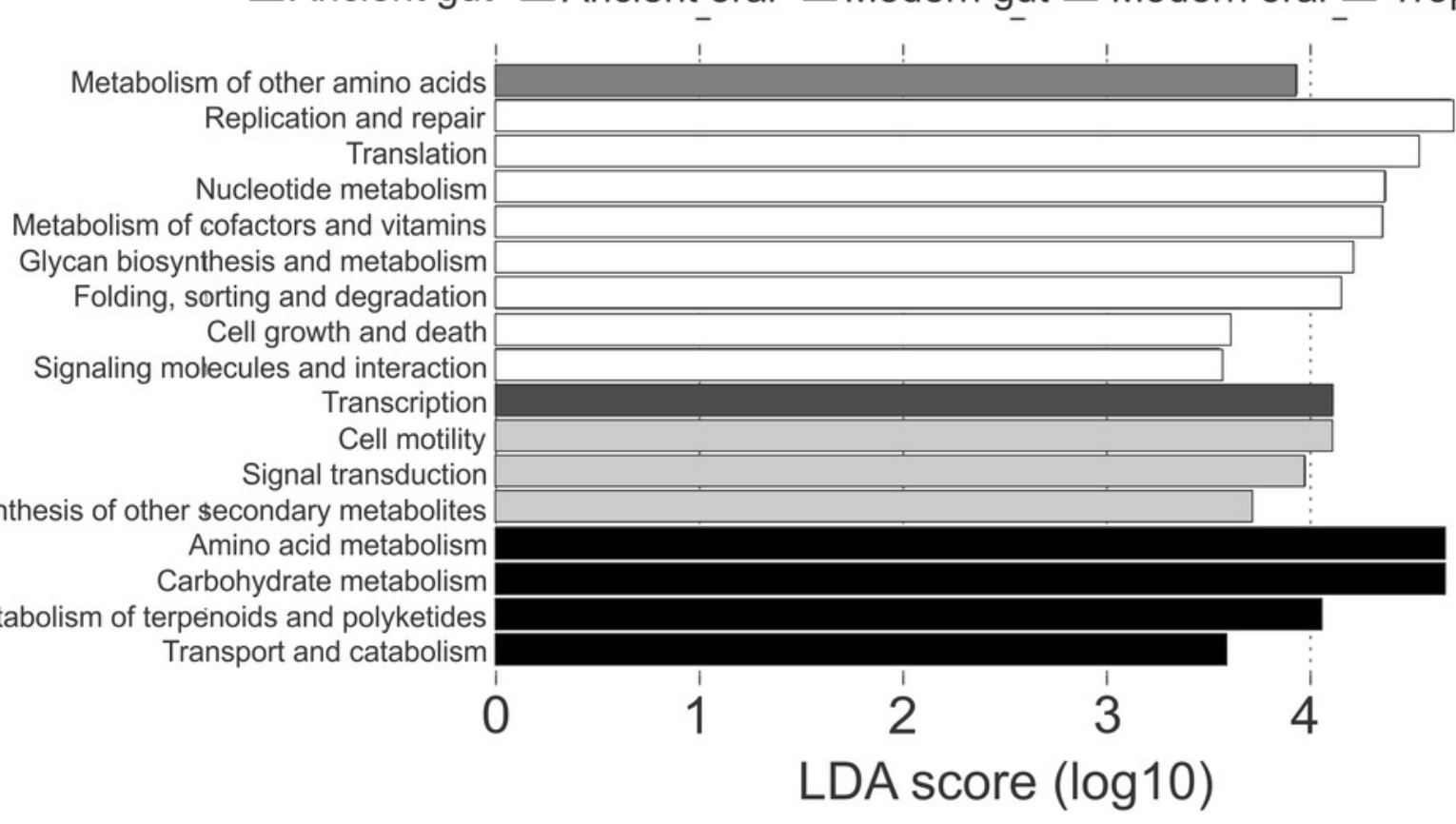




\section{Table $\mathbf{1}$ (on next page)}

Sample description

Sample description. All samples were collected from the archeological site YTA-2 at the Sorcé Estate in La Hueca, Vieques, Puerto Rico. Samples date from 10 A.D. to 385 A.D. 
1 Table 1. Sample description. All samples were collected from the archeological site YTA-2 at the Sorcé Estate in La Hueca, Vieques,

2 Puerto Rico. Samples date from 10 A.D. to 385 A.D.

\begin{tabular}{|c|c|c|c|c|c|c|c|}
\hline Sample & $\begin{array}{l}\text { Depth } \\
\text { (m) }\end{array}$ & Description & $\begin{array}{l}\text { Weight } \\
\text { (mg) }\end{array}$ & $\begin{array}{l}\text { Sequences } \\
\text { prior } \\
\text { filtering } \\
\end{array}$ & $\begin{array}{l}\text { OTUs } \\
\text { prior } \\
\text { filtering }\end{array}$ & $\begin{array}{l}\text { Sequences } \\
\text { after } \\
\text { filtering }\end{array}$ & $\begin{array}{l}\text { OTUs } \\
\text { after } \\
\text { filtering }\end{array}$ \\
\hline $\mathrm{D} 21$ & $0.60-0.80$ & Maxillary alveolar bone fragment with (6) teeth. Moderate wear. & 2.50 & 2,677 & 497 & 799 & 304 \\
\hline E19 & $0.20-0.40$ & Loose tooth with severe wear. & 2.78 & 4,309 & 571 & 1,297 & 371 \\
\hline E26 & 0.60 & Loose molar tooth with severe wear. & 3.02 & 5,933 & 490 & 604 & 288 \\
\hline F20 & 0.80 & Loose tooth with moderate wear. & 4.10 & 3,345 & 334 & 337 & 178 \\
\hline G18 & $0.40-0.60$ & Loose teeth (2) with wear & 3.89 & 14,303 & 363 & 374 & 196 \\
\hline G21 & $0.20-0.40$ & Loose molar tooth with abscess, and teeth (3). Moderate wear. & 2.92 & 24,371 & 480 & 879 & 300 \\
\hline G22 & $0.20-0.40$ & Loose tooth with enamel detachment. Moderate wear. & 4.35 & 5,227 & 707 & 1,863 & 505 \\
\hline I19 & $0.00-0.20$ & Loose teeth (2). Moderate wear. & 2.37 & 3,523 & 499 & 877 & 339 \\
\hline $\mathrm{I} 23 \mathrm{~A}$ & $0.20-0.40$ & Molar tooth and tooth. Moderate wear. & 4.28 & 2,424 & 495 & 673 & 308 \\
\hline $\mathrm{I} 23 \mathrm{~B}$ & $0.40-0.60$ & Maxillary alveolar bone fragment with (2) teeth and loose tooth. Moderate wear. & 3.17 & 2,031 & 439 & 602 & 278 \\
\hline $\mathrm{I} 24 \mathrm{~A}$ & $0.20-0.40$ & Loose tooth. Moderate wear. & 1.95 & 2,125 & 418 & 625 & 265 \\
\hline $\mathrm{I} 24 \mathrm{~B}$ & $0.60-0.80$ & Maxillary alveolar bone fragment with (3) teeth and (2) loose teeth. Moderate wear. & 2.02 & 2,321 & 376 & 602 & 228 \\
\hline F24 & 0.60 & Mandibular alveolar bone fragment with attached teeth (11). Male. Moderate wear. & 5.25 & 3,005 & 110 & 245 & 33 \\
\hline H6 & 0.70 & Infant maxillary alveolar bone fragment with fully erupted tooth and deciduous teeth (3) & 5.14 & 56,503 & 74 & 334 & 31 \\
\hline M8 & $0.00-0.20$ & Mandibular alveolar bone fragment with (4) teeth. Male. Moderate wear & 4.98 & 2,214 & 46 & 267 & 23 \\
\hline Soil* & & Archaeological site of Sorcé & - & 22,945 & 336 & - & - \\
\hline Blank* & & & - & 34,392 & 956 & - & - \\
\hline
\end{tabular}

3 Dental calculi samples highlighted in bold were attached to bone fragments that enabled the gender or approximate age determination.

$4 \quad *$ Soil and blank sequences were filtered from calculi samples for main analyses. 


\section{Table 2 (on next page)}

Group Significance

Group significance analyses of dental calculi, dental calculi (Bone), coprolites, supragingival and subgingival plaque, saliva, stool and soil from the archaeological site of Sorcé. Selected OTUs present in both dental calculus and modern oral samples are shown. Group significance analyses were performed after filtering soil and blank OTUs. 
1 Table 2. Group significance analyses of dental calculi, dental calculi (Bone), coprolites, supragingival and subgingival plaque, saliva,

2 stool and soil from the archaeological site of Sorcé. Selected OTUs present in both dental calculus and modern oral samples are

3 shown. Group significance analyses were performed after filtering soil and blank OTUs.

\begin{tabular}{|c|c|c|c|c|c|c|c|c|c|c|c|c|c|}
\hline OTU & $\begin{array}{l}\text { Test- } \\
\text { Statistic }\end{array}$ & $\mathbf{P}$ & $\begin{array}{l}\text { FDR } \\
\mathbf{P} \\
\end{array}$ & $\begin{array}{l}\text { Bonferroni } \\
\text { P } \\
\end{array}$ & Soil* & Saliva* & Coprolite* & Calculi* & $\begin{array}{l}\text { Subgingival } \\
\text { plaque* }\end{array}$ & $\begin{array}{l}\text { Calculi } \\
\text { (Bone)* }\end{array}$ & $\begin{array}{l}\text { Supragingival } \\
\text { plaque* }^{*}\end{array}$ & Stool* & Taxonomy \\
\hline 349024 & 29.790 & 0.000 & 0.000 & 1.000 & 0 & 0.000 & 0 & 0.833 & 0.000 & 0.000 & 0.250 & 32427.600 & Streptococcus sp. \\
\hline 1090059 & 26.416 & 0.000 & 0.002 & 1.000 & 0 & 3.500 & 0 & 0.250 & 1.000 & 0.000 & 2.750 & 174.400 & Granulicatella sp. \\
\hline 139056 & 5.396 & 0.612 & 0.735 & 1.000 & 0 & 0.250 & 0 & 0.250 & 0.250 & 0.000 & 0.000 & 0.000 & Aggregatibacter sp. \\
\hline 966331 & 5.396 & 0.612 & 0.735 & 1.000 & 0 & 0.000 & 0 & 0.250 & 0.250 & 0.000 & 0.250 & 0.000 & Dermacoccus sp. \\
\hline 1082294 & 36.401 & 0.000 & 0.000 & 0.087 & 0 & 72.750 & 0 & 0.167 & 18.250 & 0.000 & 207.000 & 0.000 & Streptococcus sp. \\
\hline 1065974 & 22.882 & 0.002 & 0.005 & 1.000 & 0 & 5.250 & 0 & 0.167 & 1.500 & 0.000 & 4.500 & 46.800 & Vagococcus sp. \\
\hline 131775 & 5.464 & 0.603 & 0.725 & 1.000 & 0 & 0.000 & 0 & 0.167 & 0.000 & 0.000 & 1.250 & 0.000 & Micrococcaceae sp. \\
\hline 535196 & 5.123 & 0.645 & 0.759 & 1.000 & 0 & 0.000 & 0 & 0.167 & 0.250 & 0.000 & 0.000 & 0.000 & Rhodobacteraceae sp. \\
\hline 962249 & 34.888 & 0.000 & 0.000 & 0.167 & 0 & 18.500 & 0 & 0.083 & 12.250 & 0.000 & 12.250 & 0.000 & Veillonella dispar \\
\hline 1017181 & 29.704 & 0.000 & 0.000 & 1.000 & 0 & 8.750 & 0 & 0.083 & 4.500 & 0.000 & 4.000 & 20.400 & Rothia mucilaginosa \\
\hline 1059729 & 28.524 & 0.000 & 0.001 & 1.000 & 0 & 6.500 & 0 & 0.083 & 1.000 & 0.000 & 5.000 & 4.400 & Granulicatella sp. \\
\hline 73471 & 22.635 & 0.002 & 0.006 & 1.000 & 0 & 0.000 & 0 & 0.083 & 7.750 & 0.000 & 16.250 & 1.200 & Actinomyces sp. \\
\hline 1084417 & 19.585 & 0.007 & 0.018 & 1.000 & 0 & 5.000 & 0 & 0.083 & 12.000 & 0.000 & 9.250 & 2.800 & Lautropia sp. \\
\hline 925707 & 15.381 & 0.031 & 0.065 & 1.000 & 0 & 1.000 & 0 & 0.083 & 12.750 & 0.000 & 0.750 & 2.000 & Gemellaceae sp. \\
\hline 499378 & 14.442 & 0.044 & 0.090 & 1.000 & 0 & 0.000 & 0 & 0.083 & 0.500 & 0.000 & 0.750 & 0.000 & Actinomyces sp. \\
\hline 509773 & 10.181 & 0.179 & 0.334 & 1.000 & 0 & 18.500 & 0 & 0.083 & 2.000 & 0.000 & 0.250 & 0.000 & Streptococcus sp. \\
\hline 577170 & 9.319 & 0.231 & 0.398 & 1.000 & 0 & 0.000 & 0 & 0.083 & 0.250 & 0.000 & 0.000 & 4.400 & Bacteroides sp. \\
\hline 542066 & 5.629 & 0.584 & 0.702 & 1.000 & 0 & 0.250 & 0 & 0.083 & 0.000 & 0.000 & 0.250 & 0.000 & Actinomycetales sp. \\
\hline 432284 & 5.464 & 0.603 & 0.725 & 1.000 & 0 & 0.500 & 0 & 0.083 & 0.000 & 0.000 & 0.000 & 0.000 & Streptophyta sp. \\
\hline 1005406 & 5.293 & 0.624 & 0.736 & 1.000 & 0 & 0.250 & 0 & 0.083 & 0.000 & 0.000 & 0.000 & 0.000 & Staphylococcus aureus \\
\hline 898207 & 5.293 & 0.624 & 0.736 & 1.000 & 0 & 0.250 & 0 & 0.083 & 0.000 & 0.000 & 0.000 & 0.000 & Granulicatella sp. \\
\hline 336228 & 5.293 & 0.624 & 0.736 & 1.000 & 0 & 0.250 & 0 & 0.083 & 0.000 & 0.000 & 0.000 & 0.000 & Dialister sp. \\
\hline 4301737 & 19.335 & 0.007 & 0.020 & 1.000 & 0 & 0.500 & 0 & 0.000 & 0.000 & 13.000 & 0.250 & 0.000 & Porphyromonas sp. \\
\hline 341460 & 35.743 & 0.000 & 0.000 & 0.115 & 0 & 180.750 & 0 & 0.000 & 29.500 & 0.667 & 40.500 & 0.000 & Haemophilus sp. \\
\hline 530164 & 20.981 & 0.004 & 0.011 & 1.000 & 0 & 102.750 & 0 & 0.000 & 41.000 & 0.667 & 1.000 & 0.000 & Porphyromonas sp. \\
\hline
\end{tabular}

